\title{
Pilot trial of remote monitoring to prevent malnutrition after hepatopancreatobiliary surgery
}

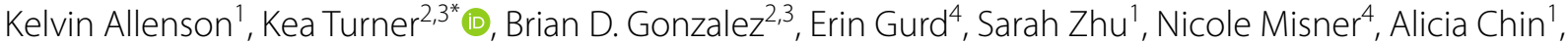 \\ Melissa Adams ${ }^{1}$, Laura Cooper', Diana Nguyen'1, Samer Naffouje ${ }^{1}$, Diana L. Castillo ${ }^{1}$, Maria Kocab³, \\ Brian James ${ }^{3}$, Jason Denbo', Jose M. Pimiento', Mokenge Malafa', Benjamin D. Powers ${ }^{1,2}$, Jason B. Fleming ${ }^{1}$, \\ Daniel A. Anaya ${ }^{1}$ and Pamela J. Hodul ${ }^{1}$
}

\begin{abstract}
Background: Patients undergoing hepatopancreatobiliary (HPB) surgery, such patients with pancreatic, periampullary, and liver cancer, are at high risk for malnutrition. Malnutrition increases surgical complications and reduces overall survival. Despite its severity, there are limited interventions addressing malnutrition after HPB surgery. The aim of this pilot trial was to examine feasibility, acceptability, usability, and preliminary efficacy of a remote nutrition monitoring intervention after HPB surgery.

Methods: Participants received tailored nutritional counseling before and after surgery at 2 and 4 weeks after hospital discharge. Participants also recorded nutritional intake daily for 30 days, and these data were reviewed remotely by registered dietitians before nutritional counseling visits. Descriptive statistics were used to describe study outcomes.

Results: All 26 patients approached to participate consented to the trial before HPB surgery. Seven were excluded after consent for failing to meet eligibility criteria (e.g., did not receive surgery). Nineteen participants (52.6\% female, median age $=65$ years) remained eligible for remote monitoring post-surgery. Nineteen used the mobile app food diary, $79 \%$ of participants recorded food intake for greater than $80 \%$ of study days, $95 \%$ met with the dietitian for all visits, and $89 \%$ were highly satisfied with the intervention. Among participants with complete data, the average percent caloric goal obtained was $82.4 \%$ (IQR: 21.7).
\end{abstract}

Conclusions: This intervention was feasible and acceptable to patients undergoing HPB surgery. Preliminary efficacy data showed most participants were able to meet calorie intake goals. Future studies should examine intervention efficacy in a larger, randomized controlled trial.

Trial registration: Clinicaltrials.gov. Registered 16 September 2019, https://clinicaltrials.gov/ct2/show/NCT04091 165. Keywords: Mobile app, Digital health, Surgery, Hepatopancreaticobiliary, Nutrition, Malnutrition, Cancer

*Correspondence: kea.turner@moffitt.org

${ }^{3}$ University of South Florida Morsani College of Medicine, Tampa, FI, USA

Full list of author information is available at the end of the article

\section{Introduction}

Hepatopancreaticobiliary (HPB) surgeries have increased over the past two decades in the U.S. [1-3]. Part of this growth has been driven by a rise in pancreatic, liver, and periampullary cancers, which are treated through HPB surgery [4-6]. HPB surgery is associated with extensive morbidity. After HPB surgery, 
about a quarter of patients experience serious complications, such as infections and blood clots [7-9], and about $15-20 \%$ of patients are readmitted to the hospital within 30 days of HPB surgery $[10,11]$. One of the most challenging aspects of patient management following HPB surgery is malnutrition, or the inadequate uptake or absorption of nutrients for maintaining one's health [12]. Up to $40 \%$ of patients undergoing HPB surgery experience malnutrition 30 days after surgery [13-16]. There is a critical need to develop interventions that reduce malnutrition among patients undergoing $\mathrm{HPB}$ surgery.

There are several reasons why malnutrition is common after HPB surgery. HPB surgeries change the digestive system, often causing symptoms that interfere with food intake and malabsorption of nutrients [17-19]. Patients can also experience complications that further interfere with nutrition, such as pancreatic exocrine insufficiency (i.e., lack of digestive enzymes), endocrine insufficiency (i.e., glucose intolerance), and delayed gastric emptying due to impaired motor function of the stomach [20-24]. Left untreated, malnutrition can have devastating consequences, such as impaired immune functioning, loss of lean body mass, increased risk for infection, decreased receipt of adjuvant therapy, and lower survival $[13,25-$ 27]. Despite its severity, there is limited evidence about how to treat malnutrition after HPB surgery [28, 29].

Nutritional counseling is effective for preventing malnutrition among patients with cancer, but this strategy has been underused among HPB surgery patients [28, 30-32]. To prevent malnutrition after surgery, patients must make significant dietary changes, such as eating small and frequent meals, and may require supplements or insulin to manage pancreatic exocrine or endocrine insufficiency. Despite the importance of dietary self-management, patients undergoing HPB surgery are often discharged from the hospital without any dietitian support and report feeling overwhelmed and underprepared to manage their nutrition [33, 34]. For example, fewer than a quarter of pancreatectomy patients receive any dietitian support post-surgery [31,35], even though this is recommended by clinical guidelines [20]. Prior studies suggest that nutrition counseling is enhanced when patients are given tools to track their food intake, an approach known as dietary self-monitoring [36-38]. Dietary self-monitoring or recording daily consumption of foods and beverages is an evidence-based approach for behavioral weight loss interventions [38]. In order for dietary self-monitoring to work, patients must adhere to daily tracking of their food intake [39]. However, self-monitoring can be burdensome and decline over time, especially when using paper food logs [40]. Therefore, digital tools may be optimal for promoting adherence to dietary self-monitoring among HPB surgery patients. However, this strategy has not been tested in HPB surgery patients.

To address this gap, this pilot trial examined the feasibility, acceptability, usability, and preliminary efficacy of an intervention to provide remote nutritional monitoring for patients after HPB surgery. The intervention combines two evidence-based approaches: 1) nutrition counseling from a dietitian delivered, and 2) dietary selfmonitoring through a mobile application (app). Findings from this pilot study could support future studies to test intervention efficacy in a larger trial and to scale up the intervention to additional patients at-risk for malnutrition should the intervention prove efficacious.

\section{Methods}

\section{Study design}

A single-arm, pilot trial was conducted at Moffitt Cancer Center in Tampa, FL.

\section{Study population}

Patients with operable malignant or pre-malignant disease of the liver or pancreas, with treatment plan for neoadjuvant chemotherapy or chemoradiation or up-front resection were prospectively identified, consented, and followed through the Pancreatic and Hepatobiliary clinics between August 2019 to May 2020. Eligible patients were 18 years or older with biopsy-proven malignancy or those with pre-malignant diagnoses undergoing planned pancreatectomy or hepatectomy. Patients were also required to own a smartphone, be willing to use a mobile app for the tracking of post-operative nutritional intake, answer nutrition-related questionnaires, and be able to speak English. Patients were excluded if they were deemed ineligible for surgery or required parenteral or enteral nutrition. Patients provided written consent to participate in the study prior to surgery. The target sample size was 20 patients, which was deemed sufficient to test feasibility. The Advarra Institutional Review Board reviewed and approved all study activities, and the study was registered at ClinicalTrials.gov (NCT04091165).

\section{Dietitian counseling and mobile app use}

Patients received dietary counseling by a registered dietitian with oncology experience in the pre-, peri-, and postoperative setting. Before surgery, patients were screened for malnutrition using a validated screening tool, the Patient-Generated Subjective Global Assessment [41]. Information from the malnutrition screening tool was used to individualize calorie goals for each patient. The dietitian set calorie goals based on the Mifflin St Jeor equation with a 1.4-1.5 activity factor depending on malnutrition status. The dietitian also met with the patient after surgery while the patient was hospitalized to review 
dietary goals and provide food intake instructions. After hospital discharge, the dietitian met with the patient at 2 and 4 weeks to provide counseling on how to increase calorie intake to meet goals (e.g., calorie-dense foods, oral nutrition supplements) and strategies for managing nutrition-related side effects.

In addition to dietary counseling, patients were provided with a mobile app, the MyPlate mobile app (Leaf Group Ltd.; Santa Monica, CA) at the initial dietitian visit. The dietitian helped patients download and enter nutrition goals into the app. Patients were asked to record all dietary intake using the app for a period of 30 days after hospital discharge. The app calculates daily caloric intake based on entered data. Entry of any food items in a day was defined as use of the app for the day. The dietitian remotely monitored patients' food intake data and used the information to guide nutrition counseling visits (e.g., provided feedback on caloric goal obtainment).

\section{Study measures}

\section{Feasibility and acceptability}

Feasibility was defined as recruitment, retention, and app use rates. The benchmark for recruitment was $\geq 50 \%$ of eligible patients consenting to participate in the study. The benchmark for retention was $\geq 70 \%$ of participants retained over the study period and $\geq 70 \%$ submitting complete food intake data for $\geq 80 \%$ of study days. Acceptability was defined as patient engagement and satisfaction with the intervention. The benchmark for patient engagement was $\geq 70 \%$ of participants attending all three dietitian visits (i.e., baseline, 2 weeks, 4 weeks). Intervention satisfaction was measured using an item assessing participants' level of agreement with the statement, "I am satisfied with using the mobile app to help follow my nutrition plan." The item was measured using a 5-point Likert scale ranging from completely disagree to completely agree. The benchmark for acceptability was $\geq 70 \%$ of participants completing agreeing with the statement.

\section{Usability}

Usability was defined as self-reported ability to learn and use the app. Usability was measured by assessing participants' level of agreement with three statements: "The mobile app was easy to learn"; "Navigating the mobile app was clear and understandable"; and "It was easy to log meals on the mobile app." The items were measured using a 5-point Likert scale ranging from completely disagree to completely agree. The benchmark for usability was $\geq 70 \%$ of participants agreeing or completing agreeing with all three statements.

\section{Caregiver involvement}

We asked participants whether their informal caregiver (e.g., spouse or family member) assisted the participant with logging their food intake.

\section{Preliminary efficacy}

Preliminary efficacy was defined as the percent of caloric goal obtained over a 30-day period. This was determined by calculating the average calories consumed per day as documented in the MyPlate Calorie Counter mobile app by the calorie goal determined by the dietitian.

\section{Statistical analyses}

Descriptive statistics were used to describe study outcomes. For categorical variables, sample size and percent are reported. For continuous variables, median and interquartile range (IQR) is reported.

\section{Results}

Sample characteristics

Of the 26 participants who consented prior to surgery, seven did not receive surgery (e.g., cancer metastasis). The median age of study $(n=19)$ participants was 65 (IQR: 15) years (Table 1). Most participants were

Table 1 Participant characteristics

\begin{tabular}{|c|c|}
\hline Characteristic & $N=19$ \\
\hline Age, median (IQR) & $65.0(15.0)$ \\
\hline \multicolumn{2}{|l|}{ Gender, n (\%) } \\
\hline Female & $10(52.6)$ \\
\hline Male & $9(47.4)$ \\
\hline \multicolumn{2}{|l|}{ Race and Ethnicity, n (\%) } \\
\hline White & $16(84.1)$ \\
\hline Non-Hispanic Black & $1(5.3)$ \\
\hline Asian & $1(5.3)$ \\
\hline Hispanic & $1(5.3)$ \\
\hline Charlson Comorbidity Index, median (IQR) & $6.0(2.0)$ \\
\hline Body Mass Index, median (IQR) & $26.2(6.8)$ \\
\hline \multicolumn{2}{|l|}{ ASA Physical Status, n (\%) } \\
\hline Score 2 & $5(26.3)$ \\
\hline Score 3 & $14(73.7)$ \\
\hline \multicolumn{2}{|l|}{ Surgery type, n (\%) } \\
\hline Hepatectomy & $4(21.1)$ \\
\hline Pancreatectomy & $15(78.9)$ \\
\hline \multicolumn{2}{|l|}{ PG-SGA Score } \\
\hline 0-1 (No immediate intervention) & $2(10.5)$ \\
\hline 2-3 (Dietitian intervention recommended) & $7(36.8)$ \\
\hline 4-8 (Dietitian intervention required) & $7(36.8)$ \\
\hline$\geq 9$ (Critical need for dietitian and clinician intervention) & $3(15.9)$ \\
\hline
\end{tabular}

IQR Interquartile range, ASA American Society of Anesthesiologists, $P G$-SGA Patient-generated Subjective Global Assessment 
White (84.1\%) and female (52.6\%). The median Charlson comorbidity score was 6 (IQR: 2). The median body mass index (BMI) was 26.2 (IQR: 6.8). Most patients received a pancreatectomy $(78.9 \%)$ while fewer underwent a hepatectomy (21.1\%). PG-SGA scores measuring malnutrition risk ranged from 0 to 16 . Most patients received a score of 2-3 (36.8\%) indicating a recommendation for dietitian intervention or a score of $4-8(36.8 \%)$ indicating a dietitian intervention is required. Some patients (15.9\%) received a score of $\geq 9$ indicating a critical need for intervention while fewer patients $(10.5 \%)$ received a score of $0-1$ indicating no immediate intervention was needed. All patients indicated that they had used a mobile application previously.

\section{Feasibility and acceptability}

Of the 26 patients approached to participate in the study, all 26 consented to participate, a 100\% recruitment rate. Seven participants were excluded after consent for failing to meet inclusion criteria including 1) surgery was aborted due to intra-operative finding of metastatic disease or cirrhosis precluding safe resection or 2) patient required parenteral or enteral nutrition after surgery. The remaining eligible 19 patients were retained over the study period, a $100 \%$ retention rate. The percent of patients that submitted food intake data for at least $80 \%$ of study days was $15 / 19$ (78.9\%). The percent of patients who attended all three dietitian visits was 18/19 (94.7\%). Nearly all participants who completed the exit survey
$16 / 18$ (88.9\%) completely agreed with the statement that they were satisfied with the mobile app.

\section{Usability}

Among participants who provided survey data, most completely agreed $13 / 18(72.2 \%)$ that the app was easy to learn. Most completely agreed 12/18 (66.7\%) that navigating the app was clear and understandable. About half completely agreed 10/18 (55.6\%) that logging food through the app was easy. A few participants noted challenges with using the app, such as difficulty finding specific food items or not having enough food choices in the app.

\section{Informal caregiver involvement}

Among participants who provided survey data, a little more than a quarter $5 / 18(27.7 \%)$ reported that their informal caregiver assisted them with logging their food intake.

\section{Percent of caloric goal obtained}

Among participants with complete data $(n=15)$ (24/30 days of data), the percent caloric goal obtained was $82.4 \%$ (IQR: 21.7) over the 30-day period. During week 1 , among participants with complete data $(n=17)$ (5/7 days of data), the percent caloric goal obtained was 51.8\% (IQR: 26.5) (Fig. 1). During week 2, among participants with complete data $(n=15)$, the percent caloric goal obtained was $84.0 \%$ (IQR: 34.4 ). During week 3,

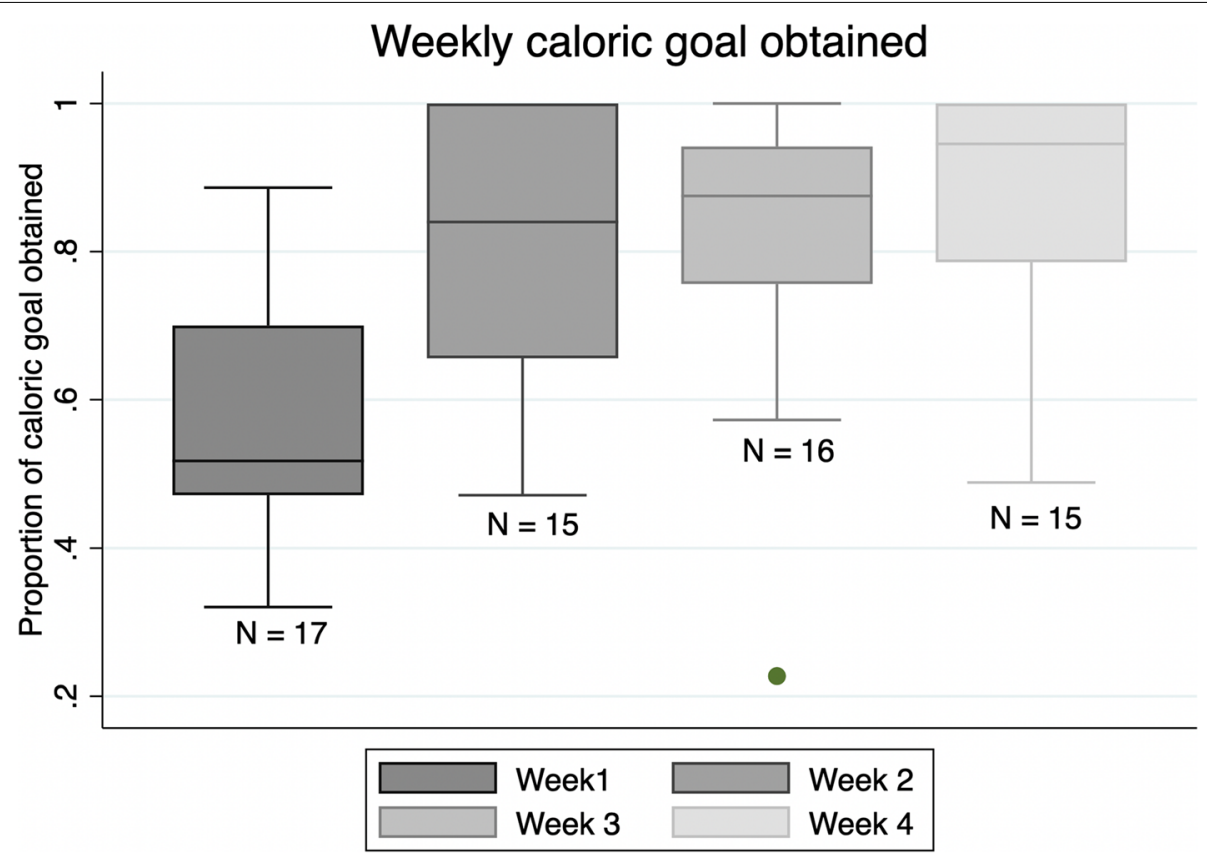

Fig. 1 Weekly caloric goal attainment among study participants 
among participants with complete data $(n=16)$, the percent caloric goal obtained was $87.5 \%$ (IQR: 18.6). During week 4 , among participants with complete data $(n=15)$, the percent caloric goal obtained was 94.5\% (IQR: 24.0).

\section{Discussion}

Overall, the goal of this study was to assess the feasibility, acceptability, usability, and preliminary efficacy of a remote nutrition counseling intervention to enhance nutrition recovery after HPB surgery. Our study found that the intervention met a priori feasibility and acceptability benchmarks. Most participants were retained over the study period, were highly engaged with the mobile app and the dietitian visits, and most reported high satisfaction with the intervention. Most participants found the app easy-to-use; however, a few participants reported challenges with locating food items within the app. In terms of preliminary efficacy, participants struggled meeting their daily caloric goals during the first week after surgery; however, most participants began achieving their caloric goals by week 2 and were able to maintain their caloric goals through weeks 3 and 4 .

Prior studies suggest that the transition from hospital to home after pancreatectomy and hepatectomy is challenging for many patients with cancer [33, 34, 42]. Patients are expected to make complex dietary changes (e.g., tracking calorie intake) and modify behavior (e.g., small, frequent meals). Few patients receive support, however, for managing these changes. The current study adds to the literature by suggesting that nutrition counseling coupled with digital tools, such as a mobile app to track food intake, is feasible and acceptable among this patient population. For example, most patients (79\%) logged food intake for more than $80 \%$ of study days and nearly all participants (95\%) attended all three dietitian visits during the study period. These findings suggest that there is patient demand for enhanced nutrition monitoring after HPB surgery. However, there are no published accounts of mobile app monitoring of post-operative caloric intake. This study is the first to our knowledge to use mobile apps for this purpose.

One of the key challenges of delivering a digital intervention is developing a tool that is easy to learn and use across a wide array of patients. Our study found that most but not all patients agreed the app was easy to learn, navigate, and use for logging food intake. Several challenges were also identified, such as difficulty finding specific food items or not having enough food choices within the app. Our dietitian noted that several participants reported challenges when the exact food item (e.g., specific brand of yogurt) was unavailable in the app. The dietitian clarified to patients that an approximation (e.g., selecting a brand of yogurt that is available) is sufficient.
In addition to optimizing patient instructions, future studies may need to assess digital literacy (e.g., eHealth literacy scale) [43] to determine whether usability varies based on pre-existing digital literacy. Our study found that about a quarter of patients required informal caregiver assistance to help enter food intake data in the mobile app. Future studies might add an informal caregiver training component to help informal caregivers also learn how to use the app.

Our study found that caloric intake trended upwards over the 30-day period. Most participants struggled with food intake in the first week post-discharge but quickly recovered during weeks 2-4. Our intervention started dietitian visits 2 weeks post-surgery. Future studies might consider starting dietitian visits 1 week after surgery, when patients have the most difficulty meeting their caloric intake goals. The increase in caloric intake over time may also be explained by the type of surgeries examined. For example, at the time of first surgical follow-up, most patients undergoing post-Whipple/total pancreatectomy advance from a low-fat diet to a regular diet.

Remote monitoring of caloric intake has the potential to be used as a clinical indicator for HPB surgery patients, which can be intervened upon via phone consultation, secure messaging or expedited clinic follow up [44-47]. Highlighting this potential, 2 of 4 patients in our study with less than $80 \%$ app compliance presented back to the hospital with significant complications including post-operative pancreatic hemorrhage and sepsis, respectively. Implementation of monitoring and counseling has the potential to extend the reach of dietitians amongst a population who many believe should receive universal nutritional screening [48, 49]. While more research is necessary, nutrition monitoring mobile apps have potential for both the pre-habilitation and post-operative setting for cancer patients and other patients receiving complex surgeries. Nutritionally replete patients benefit from fewer perioperative complications, shorter hospitalizations, higher completion of multimodality therapy, and improved survival $[50,51]$.

\section{Limitations}

This study has several limitations. First, the study was conducted at an National Cancer Institute Designated Comprehensive Cancer Center, which may have resources that are not available in all oncology settings (e.g., onsite registered dietitian). Second, this study was designed to assess feasibility, acceptability, and app usability and was not powered to evaluate efficacy. A larger trial is needed to determine the efficacy of the intervention. Findings from this study suggest that the intervention is feasible and could be tested in a larger trial. Third, the study was limited to participants who speak English. 
The usability of the app may differ for languages other than English. Future studies should test the usability of the mobile app in Spanish speakers and other languages (e.g., Arabic) available through the MyPlate app [52].

\section{Conclusion}

Malnutrition is a common and devastating consequence of cancer and surgical treatment, such as HPB surgery. Despite its severity, there are limited interventions addressing malnutrition after surgery. Patient dietary self-monitoring through a mobile app, combined with dietitian counseling, in the post-operative period after HPB surgery appears to be feasible and acceptable. Most patients were able to achieve their caloric goals within 2-4weeks of surgery, suggesting this intervention could improve patient outcomes. Further studies are needed to test this approach in a larger efficacy trial.

\section{Abbreviations}

HPB: Hepatopancreaticobiliary; IQR: Interquartile range.

\section{Acknowledgements}

N/A

\section{Authors' contributions}

Conceptualization: PJH. Investigation: KA, EG, SZ, NM, AC, MA, LC, DN, SN, DLC, $M K, B J, J D, J M P, M M, B D P, J B F, D A A, P J H$. Analysis and interpretation of data: KA, $K T, B D G, P J H$. Writing, reviewing, and editing of manuscript: KA, KT, BDG, PJH. All authors have read and approved the final manuscript.

\section{Funding}

This research was supported by internal funding from Moffitt Cancer Center. The funding bodies played no role in the design of the study and collection, analysis, and interpretation of data and in writing the manuscript.

\section{Availability of data and materials}

The authors will make de-identified data available upon request with the establishment of a data sharing agreement. Requests can be sent to kea. turner@moffitt.org.

\section{Declarations}

Ethics approval and consent to participate

This research was approved by the Advarra Institutional Review Board. Patients provided written consent to participate in the study.

\section{Consent for publication}

Not Applicable.

\section{Competing interests}

Dr. Gonzalez has received consulting fees from SureMed Compliance and KemPharm. Dr. Gonzalez participates on an advisory board for EllyHealth. Dr. Hodul has served on a speaker bureau for Abbvie. The other authors do not have any conflicts of interest to disclose.

\section{Author details}

'Department of Gastrointestinal Oncology, Moffitt Cancer Center, Tampa, Fl, USA. ${ }^{2}$ Department of Health Outcomes and Behavior, Moffitt Cancer Center, Tampa, FL, USA. ${ }^{3}$ University of South Florida Morsani College of Medicine, Tampa, FI, USA. ${ }^{4}$ Department of Nutrition Therapy, Moffitt Cancer Center, Tampa, Fl, USA.
Received: 5 September 2021 Accepted: 25 November 2021

Published online: 09 December 2021

\section{References}

1. Ali N, O'Rourke C, El-Hayek K, Chalikonda S, Jeyarajah DR, Walsh RM. Estimating the need for hepato-pancreatico-biliary surgeons in the USA. HPB (Oxford). 2015;17(4):352-6.

2. Dimick JB, Wainess RM, Cowan JA, Upchurch GR, Knol JA, Colletti LM. National trends in the use and outcomes of hepatic resection. J Am Coll Surg. 2004;199(1):31-8.

3. Nathan H, Segev DL, Mayo SC, Choti MA, Cameron AM, Wolfgang CL, et al. National trends in surgical procedures for hepatocellular carcinoma: 1998-2008. Cancer. 2012;118(7):1838-44.

4. Gordon-Dseagu VL, Devesa SS, Goggins M, Stolzenberg-Solomon R. Pancreatic cancer incidence trends: evidence from the Surveillance Epidemiology and End Results (SEER) population-based data. Int J Epidemiol. 2018;47(2):427-39.

5. Liu Z, Jiang Y, Yuan H, Fang Q, Cai N, Suo C, et al. The trends in incidence of primary liver cancer caused by specific etiologies: results from the Global Burden of Disease Study 2016 and implications for liver cancer prevention. J Hepatol. 2019;70(4):674-83.

6. Shi HY, Wang SN, Lee KT. Temporal trends and volume-outcome associations in periampullary cancer patients: a propensity score-adjusted nationwide population-based study. Am J Surg. 2014;207(4):512-9.

7. Lucas DJ, Sweeney JF, Pawlik TM. The timing of complications impacts risk of readmission after hepatopancreatobiliary surgery. Surgery. 2014;155(5):945-53

8. Kneuertz PJ, Pitt HA, Bilimoria KY, Smiley JP, Cohen ME, Ko CY, et al. Risk of morbidity and mortality following hepato-pancreato-biliary surgery. J Gastrointest Surg. 2012;16(9):1727-35.

9. Chen Q, Beal EW, Kimbrough CW, Bagante F, Merath K, Dillhoff M, et al. Perioperative complications and the cost of rescue or failure to rescue in hepato-pancreato-biliary surgery. HPB (Oxford). 2018;20(9):854-64.

10. Reddy DM, Townsend CM, Kuo YF, Freeman JL, Goodwin JS, Riall TS. Readmission after pancreatectomy for pancreatic cancer in Medicare patients. J Gastrointest Surg. 2009;13(11):1963-74 discussion 1974-1965.

11. Schneider EB, Hyder O, Wolfgang CL, Hirose K, Choti MA, Makary MA, et al. Patient readmission and mortality after surgery for hepato-pancreatobiliary malignancies. J Am Coll Surg. 2012;215(5):607-15.

12. Aotani N, Yasui-Yamada S, Kagiya N, Takimoto M, Oiwa Y, Matsubara A et al. Malnutrition by European Society for Clinical Nutrition and Metabolism criteria predicts prognosis in patients with gastrointestinal and hepatobiliary-pancreatic cancer. Clin Nutr ESPEN. 2021;42:265-71.

13. La Torre M, Ziparo V, Nigri G, Cavallini M, Balducci G, Ramacciato G. Malnutrition and pancreatic surgery: prevalence and outcomes. J Surg Oncol. 2013;107(7):702-8.

14. Morita Y, Sakaguchi T, Kitajima R, Furuhashi S, Kiuchi R, Takeda M, et al. Body weight loss after surgery affects the continuity of adjuvant chemotherapy for pancreatic cancer. BMC Cancer. 2019;19(1):416.

15. Pausch T, Hartwig W, Hinz U, Swolana T, Bundy BD, Hackert T, et al. Cachexia but not obesity worsens the postoperative outcome after pancreatoduodenectomy in pancreatic cancer. Surgery. 2012;152(3 Suppl 1):S81-8.

16. Huang TH, Hsieh CC, Kuo LM, Chang CC, Chen CH, Chi CC, et al. Malnutrition associated with an increased risk of postoperative complications following hepatectomy in patients with hepatocellular carcinoma. HPB (Oxford). 2019;21(9):1150-5.

17. Heerkens HD, Tseng DS, Lips IM, van Santvoort HC, Vriens MR, Hagendoorn J, et al. Health-related quality of life after pancreatic resection for malignancy. Br J Surg. 2016;103(3):257-66.

18. Crippa S, Domínguez I, Rodríguez JR, Razo O, Thayer SP, Ryan DP, et al. Quality of life in pancreatic cancer: analysis by stage and treatment. J Gastrointest Surg. 2008;12(5):783-93 discussion 793-784.

19. van Dijk SM, Heerkens HD, Tseng DSJ, Intven M, Molenaar IQ, van Santvoort HC. Systematic review on the impact of pancreatoduodenectomy on quality of life in patients with pancreatic cancer. HPB (Oxford). 2018;20(3):204-15.

20. Gianotti L, Besselink MG, Sandini M, Hackert T, Conlon K, Gerritsen A, et al. Nutritional support and therapy in pancreatic surgery: a position paper 
of the International Study Group on Pancreatic Surgery (ISGPS). Surgery. 2018;164(5):1035-48.

21. Lim PW, Dinh KH, Sullivan M, Wassef WY, Zivny J, Whalen GF, et al. Thirtyday outcomes underestimate endocrine and exocrine insufficiency after pancreatic resection. HPB (Oxford). 2016;18(4):360-6.

22. Slezak $L A$, Andersen DK. Pancreatic resection: effects on glucose metabolism. World J Surg. 2001;25(4):452-60.

23. Scavini M, Dugnani E, Pasquale V, Liberati D, Aleotti F, Di Terlizzi G, et al. Diabetes after pancreatic surgery: novel issues. Curr Diab Rep. 2015;15(4):16

24. Panwar R, Pal S. The International Study Group of Pancreatic Surgery definition of delayed gastric emptying and the effects of various surgical modifications on the occurrence of delayed gastric emptying after pancreatoduodenectomy. Hepatobiliary Pancreat Dis Int. 2017;16(4):353-63.

25. Karagianni VT, Papalois AE, Triantafillidis JK. Nutritional status and nutritional support before and after pancreatectomy for pancreatic cancer and chronic pancreatitis. Indian J Surg Oncol. 2012;3(4):348-59.

26. Dewys WD, Begg C, Lavin PT, Band PR, Bennett JM, Bertino JR, et al. Prognostic effect of weight loss prior tochemotherapy in cancer patients. Am J Med. 1980;69(4):491-7.

27. Fearon K, Arends J, Baracos V. Understanding the mechanisms and treatment options in cancer cachexia. Nat Rev Clin Oncol. 2013:10(2):90.

28. Walsh D, Szafranski M, Aktas A, Kadakia KC. Malnutrition in cancer care: time to address the elephant in the room. J Oncol Pract. 2019;15(7):357-9.

29. Trujillo EB, Claghorn K, Dixon SW, Hill EB, Braun A, Lipinski E, et al. Inadequate nutrition coverage in outpatient cancer centers: results of a national survey. J Oncol. 2019;2019:7462940.

30. Lee JLC, Leong LP, Lim SL. Nutrition intervention approaches to reduce malnutrition in oncology patients: a systematic review. Support Care Cancer. 2016:24(1):469-80.

31. Nemer L, Krishna SG, Shah ZK, Conwell DL, Cruz-Monserrate Z, Dillhoff $M$, et al. Predictors of pancreatic cancer-associated weight loss and nutritional interventions. Pancreas. 2017;46(9):1152-7.

32. Beesley VL, Janda M, Goldstein D, Gooden H, Merrett ND, O'Connell $\mathrm{DL}$, et al. A tsunami of unmet needs: pancreatic and ampullary cancer patients' supportive care needs and use of community and allied health services. Psychooncology. 2016;25(2):150-7.

33. Dunleavy L, Al-Mukhtar A, Halliday V. Pancreatic enzyme replacement therapy following surgery for pancreatic cancer: an exploration of patient self-management. Clin Nutr ESPEN. 2018;26:97-103.

34. Cooper C, Burden ST, Molassiotis A. An explorative study of the views and experiences of food and weight loss in patients with operable pancreatic cancer perioperatively and following surgical intervention. Support Care Cancer. 2015;23(4):1025-33.

35. Sikkens EC, Cahen DL, van Eijck C, Kuipers EJ, Bruno MJ. The daily practice of pancreatic enzyme replacement therapy after pancreatic surgery: a northern European survey: enzyme replacement after surgery. J Gastrointest Surg. 2012;16(8):1487-92.

36. Patel ML, Hopkins CM, Brooks TL, Bennett GG. Comparing self-monitoring strategies for weight loss in a smartphone app: randomized controlled trial. JMIR Mhealth Uhealth. 2019;7(2):e12209.

37. Stephens JD, Yager AM, Allen J. Smartphone technology and text messaging for weight loss in young adults: a randomized controlled trial. J Cardiovasc Nurs. 2017:32(1):39-46.

38. Butryn ML, Webb V, Wadden TA. Behavioral treatment of obesity. Psychiatr Clin North Am. 2011;34(4):841-59.

39. Burke LE, Wang J, Sevick MA. Self-monitoring in weight loss: a systematic review of the literature. J Am Diet Assoc. 2011;111(1):92-102.

40. Carter MC, Burley VJ, Nykjaer C, Cade JE. Adherence to a smartphone application for weight loss compared to website and paper diary: pilot randomized controlled trial. J Med Internet Res. 2013:15(4):e32.

41. Bauer J, Capra S, Ferguson M. Use of the scored Patient-Generated Subjective Global Assessment (PG-SGA) as a nutrition assessment tool in patients with cancer. Eur J Clin Nutr. 2002;56(8):779-85.

42. Wong SS, George TJ, Godfrey M, Le J, Pereira DB. Using photography to explore psychological distress in patients with pancreatic cancer and their caregivers: a qualitative study. Support Care Cancer. 2019;27(1):321-8

43. Norman CD, Skinner HA. eHEALS: the eHealth literacy scale. J Med Internet Res. 2006:8(4):e27.
44. Gustavell T, Sundberg K, Segersvärd R, Wengström Y, Langius-Eklöf A. Decreased symptom burden following surgery due to support from an interactive app for symptom management for patients with pancreatic and periampullary cancer. Acta Oncol. 2019;58(9):1307-14.

45. Hanna L, Huggins CE, Furness K, Silvers MA, Savva J, Frawley H, et al. Effect of early and intensive nutrition care, delivered via telephone or mobile application, on quality of life in people with upper gastrointestinal cancer: study protocol of a randomised controlled trial. BMC Cancer. 2018:18(1):1-13.

46. Semple JL, Sharpe S, Murnaghan ML, Theodoropoulos J, Metcalfe KA. Using a mobile app for monitoring post-operative quality of recovery of patients at home: a feasibility study. JMIR mHealth uHealth. 2015;3(1):e18.

47. Goyal S, Cafazzo JA. Mobile phone health apps for diabetes management: current evidence and future developments. QJM: Int J Med. 2013;106(12):1067-9.

48. Caccialanza R, Lobascio F, Brugnatelli S, Pedrazzoli P. Nutritional support in pancreatic cancer. Cancer. 2020;126(8):1810-1.

49. Moffat GT, Epstein AS, O'Reilly EM. Pancreatic cancer-a disease in need: optimizing and integrating supportive care. Cancer. 2019:125(22):3927-35.

50. Andreyev H, Norman A, Oates J, Cunningham D. Why do patients with weight loss have a worse outcome when undergoing chemotherapy for gastrointestinal malignancies? Eur J Cancer. 1998;34(4):503-9.

51. Ovesen L, Allingstrup L, Hannibal J, Mortensen EL, Hansen OP. Effect of dietary counseling on food intake, body weight, response rate, survival, and quality of life in cancer patients undergoing chemotherapy: a prospective, randomized study. J Clin Oncol. 1993;11(10):2043-9.

52. MyPlate [https://www.myplate.gov/resources/graphics/myplate-graph ics]. Accessed 7 Dec 2021.

\section{Publisher's Note}

Springer Nature remains neutral with regard to jurisdictional claims in published maps and institutional affiliations.

Ready to submit your research? Choose BMC and benefit from:

- fast, convenient online submission

- thorough peer review by experienced researchers in your field

- rapid publication on acceptance

- support for research data, including large and complex data types

- gold Open Access which fosters wider collaboration and increased citations

- maximum visibility for your research: over $100 \mathrm{M}$ website views per year

At BMC, research is always in progress.

Learn more biomedcentral.com/submissions 